\title{
A NOTE ON DISCRIMINANTAL ARRANGEMENTS
}

\author{
MICHAEL FALK
}

(Communicated by Jeffry N. Kahn)

\begin{abstract}
Let $\mathscr{A}_{0}$ be a fixed affine arrangement of $n$ hyperplanes in general position in $\mathbf{K}^{k}$. Let $U(n, k)$ denote the set of general position arrangements whose elements are parallel translates of the hyperplanes of $\mathscr{A}_{0}$. Then $U(n, k)$ is the complement of a central arrangement $\mathscr{B}(n, k)$. These are the well-known discriminantal arrangements introduced by Y. I. Manin and V. V. Schechtman. In this note we give an explicit description of $\mathscr{B}(n, k)$ in terms of the original arrangement $\mathscr{A}_{0}$. In terms of the underlying matroids, $\mathscr{B}(n, k)$ realizes an adjoint of the dual of the matroid associated with $\mathscr{A}_{0}$. Using this description we show that, contrary to the conventional wisdom, neither the intersection lattice of $\mathscr{B}(n, k)$ nor the topology of $U(n, k)$ is independent of the original arrangement $\mathscr{A}_{0}$.
\end{abstract}

\section{INTRODUCTION}

Let $\mathbf{K}$ be a field. An (affine) arrangement $\mathscr{A}$ over $\mathbf{K}$ is a finite set of affine hyperplanes in $\mathbf{K}^{k}$. If the hyperplanes of $\mathscr{A}$ all pass through the origin, then $\mathscr{A}$ is central. The intersection lattice of a central arrangement $\mathscr{A}$ is the poset $\{\bigcap \mathscr{S} \mid \mathscr{S} \subseteq \mathscr{A}\}$, ordered by reverse inclusion. The complement of $\mathscr{A}$ is $\mathbf{K}^{k} \backslash \cup \mathscr{A}$. We say $\mathscr{A}$ is a general position arrangement if the intersection of any $p$ hyperplanes of $\mathscr{A}$ has codimension $p$, if $p \leq k$, and is empty if $p>k$.

Let $\mathscr{A}_{0}$ be a fixed general position arrangement of $n$ hyperplanes. In [6] Y. I. Manin and V. V. Schechtman initiated the study of the space $U(n, k)$ of general position arrangements whose hyperplanes are parallel to those of $\mathscr{A}_{0}$. By choosing defining equations for the elements of $\mathscr{A}_{0}$ this space may be identified with a subspace of $\mathbf{K}^{n}$. It turns out that $U(n, k)$ is itself the complement of a central arrangement of hyperplanes $\mathscr{B}(n, k)$ in $\mathbf{K}^{n}$. In [6] these arrangements are called discriminantal arrangements; they are often referred to as Manin-Schechtman arrangements. In case $k=1, U(n, k)$ is

Received by the editors September 29, 1992 and, in revised form, March 23, 1993.

1991 Mathematics Subject Classification. Primary 52B30, 05B35, 52B40.

Key words and phrases. Manin-Schechtman arrangement, Grassmann stratum, dual matroid, adjoint.

The author was partially supported by NSF grant DMS-9004202 and NAU Organized Research Program. 
the configuration space of $n$ distinct points in $\mathbf{K}$; the arrangement $\mathscr{B}(n, 1)$ is the braid arrangement consisting of the hyperplanes $x_{i}=x_{j}, 1 \leq i<j \leq n$.

It is frequently stated (see [7], [8], or [5], for instance) that the intersection lattice of $\mathscr{B}(n, k)$ and/or the topology of $U(n, k)$ is independent of the arrangement $\mathscr{A}_{0}$, and depends only on $n$ and $k$. Indeed this is not the case. The purpose of this note is to provide a counter-example and to give a simple and precise description of the dependence of $\mathscr{B}(n, k)$ on $\mathscr{A}_{0}$.

The authors of [6] apparently did not intend to make any claim of independence. In that paper appears the following caveat:

However, we shall be concerned mostly with its combinatorial invariants which are constant on an open Zariski dense subset of all $n$-arrangements in $F^{n}$ [sic]. Stating properties of such invariants we shall tacitly assume that our arrangements $\left(H_{i}^{0}\right)$ are general in this sense.

On the other hand, this vague hypothesis plays no role in the proof of the Proposition 4 of [6], which is intended to give a partial description of the intersection lattice of $\mathscr{B}(n, k)$. Although the assertions made in the proof of that proposition are correct, the conclusion they are meant to imply is false in general (see remarks following Example 3.2). Indeed, "generic" in the sense quoted above must be taken to mean "Proposition 4(c) holds".

So if $\mathscr{A}_{0}$ is sufficiently general, the description of the intersection lattice of $\mathscr{B}(n, k)$ given in [6] is correct. In [7] it was observed that, for $n=k+3$, this lattice agrees with that of a central arrangement $\mathscr{B}$ formed as follows: in $\mathbf{K}^{3}$ with coordinates $\left(x_{1}, x_{2}, x_{3}\right)$ choose $n$ points in general position in the plane $x_{3}=1$, with the additional property that the $\left(\begin{array}{l}n \\ 2\end{array}\right)$ lines determined by them contain no parallels. Then $\mathscr{B}$ consists of the $\left(\begin{array}{l}n \\ 2\end{array}\right)$ planes through the origin spanned by these lines. We give a similar but more precise description, with no restrictions on $n$ and $k$, which also clarifies somewhat the meaning of "sufficiently general". We will construct a set of $n$ points in general position in $\mathbf{K}^{n-k}$ such that the set of $\left(\begin{array}{c}n \\ k-1\end{array}\right)$ hyperplanes they span is precisely the "essential part" of $\mathscr{B}(n, k)$.

This simplification is made possible using an (indirect) identification of the arrangements in question with points of the Grassmannian $\mathscr{G}(n+1, k+1)$. Here $\mathscr{G}(p, q)$ refers to the set of $q$-dimensional linear subspaces of $\mathbf{K}^{p}$. This approach is developed in section 2. In section 3 we construct an example of an arrangement which is not "general" in the sense of [6]. Our example also shows that the Manin-Schechtman construction fails to yield a fibering of the open stratum in the matroid stratification of the Grassmannian [3, 2].

\section{2. $U(n, k)$ IN THE GENERIC GRASSMANN STRATUM}

The rank of a central arrangement $\mathscr{A}$ in $\mathbf{K}^{k}$ is the codimension of $\cap A$. If $\mathscr{A}$ has rank $k$, we say $\mathscr{A}$ is essential. In any case the natural projection will yield an essential arrangement $\mathscr{A}_{\text {ess }}$ in $\mathbf{K}^{k} / \cap \mathscr{A}$, which we will call the essential part of $\mathscr{A}$. The intersection lattice of $\mathscr{A}_{\text {ess }}$ agrees with that of $\mathscr{A}$ and their complements differ by a direct factor of $\mathbf{K}^{k-r}$, where $r$ is the rank of $\mathscr{A}$. In the terminology of [7], $\mathscr{A}$ is the product of $\mathscr{A}_{\text {ess }}$ with the empty arrangement 
in $\mathbf{K}^{k-r}$. The discriminantal arrangement $\mathscr{B}(n, k)$ in $\mathbf{K}^{n}$ has rank $n-k$. It will be quite trivial to see the $\mathbf{K}^{k}$ factor of $U(n, k)$ in our picture.

In order to formulate our main theorem we must describe the relation between arrangements and Grassmann manifolds. Let $\mathscr{A}=\left\{H_{1}, \ldots, H_{n}\right\}$ be an essential central arrangement. Choose linear forms $\phi_{i}: \mathbf{K}^{k} \rightarrow \mathbf{K}$ with $H_{i}=\operatorname{kernel}\left(\phi_{i}\right)$. Let $P=P(\mathscr{A})$ be the image of the linear map $\left(\phi_{1}, \ldots, \phi_{n}\right): \mathbf{K}^{k} \rightarrow \mathbf{K}^{n}$. Then $P$ is a linear subspace of $\mathbf{K}^{n}$ of dimension $k$, i.e., a point of $\mathscr{G}(n, k)$. This is a slight abuse of notation, since $P(\mathscr{A})$ actually depends on the choice of defining forms. Different choices yield points in the $\left(\mathbf{C}^{*}\right)^{n}$-orbit of $P$ in $\mathscr{G}(n, k)$.

A copy of the arrangement $\mathscr{A}$ inside $P$ is formed by intersecting $P$ with the coordinate hyperplanes of $\mathbf{K}^{n}$. We say $\mathscr{A}$ and $\mathscr{A}^{\prime}$ are linearly equivalent arrangements if some linear change of coordinates in $\mathbf{K}^{k}$ carries the defining forms of $\mathscr{A}$ to those of $\mathscr{A}^{\prime}$. Then $P(\mathscr{A})=P\left(\mathscr{A}^{\prime}\right)$ if and only if $\mathscr{A}$ and $\mathscr{A}^{\prime}$ are linearly equivalent. In particular, given $P \in \mathscr{G}(n, k)$ satisfying some transversality conditions, one can recover an arrangement $\mathscr{A}$ (more precisely, the forms $\left.\phi_{i}\right)$ with $P=P(\mathscr{A})$ once a basis for $P$ is chosen.

For $I \subseteq\{1, \ldots, n\}$ let $\xi_{I}$ denote the coordinate subspace $\left\{x \mid x_{i}=0\right.$ for $i \in I\}$ in $\mathbf{K}^{k}$. Note that the codimension of $\xi_{I}$ in $\mathbf{K}^{k}$ is $|I|$. Thus the codimension of $P \cap \xi_{I}$ in $P$ is at most $|I|$.

Henceforth assume $k \geq 2$. Given an affine arrangement $\mathscr{A}$ we denote by $\overline{\mathscr{A}}$ the central arrangement obtained by translating the hyperplanes of $\mathscr{A}$ back to the origin. Let $\mathscr{A}_{0}$ be a general position arrangement in $\mathbf{K}^{k}$. Let $P=P\left(\overline{\mathscr{A}_{0}}\right)$ and let $Q$ be the orthogonal complement of $P$ in $\mathbf{K}^{n}$. Choose a basis $\left\{v_{1}, \ldots, v_{\ell}\right\}$ for $Q, \ell=n-k$, and write $v_{i}=\left(v_{i 1}, \ldots, v_{i n}\right)$. Let $w_{j}=\left(v_{1 j}, \ldots, v_{\ell j}\right) \in \mathbf{K}^{\ell}$, for $1 \leq j \leq n$.

Proposition 2.1. The set $\left\{w_{1}, \ldots, w_{n}\right\}$ is in general position in $\mathbf{K}^{\ell}$.

Proof. Since $\mathscr{A}_{0}$ is a general position arrangement, $\overline{\mathscr{A}}_{0}$ has the property that $\bigcap \mathscr{S}=0$ for all $\mathscr{S} \subseteq \overline{\mathscr{A}_{0}}$ with $|\mathscr{S}|=k$. Also, $H, H^{\prime} \in \mathscr{A}_{0}$ implies $H \cap H^{\prime} \neq \varnothing$, so the hyperplanes of $\mathscr{A}_{0}$ translate to $n$ distinct hyperplanes in $\overline{\mathscr{A}_{0}}$. Then $P \cap \xi_{I}=0$ for all $|I|=k$. Now $P$ is defined by $\sum_{j=1}^{n} v_{i j} x_{j}=0$ for $1 \leq i \leq \ell$, and $P \cap \xi_{I}$ is defined by setting $x_{i}=0$ for $i \in I$ in this system. Thus we see that all $\ell \times \ell$ minors of $\left[v_{i j}\right]$ are nonzero. This in turn implies the result, that any $\ell$ of the $w_{j}$ form a basis of $\mathbf{K}^{\ell}$.

We will call a central arrangement in $\mathbf{K}^{k}$ generic if every collection of $k$ hyperplanes intersects in the origin. The corresponding set $\Gamma(n, k)=\{P \in$ $\mathscr{G}(n, k) \mid P \cap \xi_{I}=0$ for $\left.|I|=k\right\}$ is the unique open stratum in the matroid stratification of $\mathscr{G}(n, k)[3,2]$. We call $\Gamma(n, k)$ the generic stratum of $\mathscr{G}(n, k)$. As in the proof of $2.1, \mathscr{A}$ general position implies $P(\overline{\mathscr{A}}) \in \Gamma(n, k)$ for $n=|\mathscr{A}|$ and $k \geq 2$.

We can now state our main theorem.

Theorem 2.2. Let $\mathscr{B}(n, k)$ be the discriminantal arrangement associated with the general position arrangement $\mathscr{A}_{0}$. Then the essential part of $\mathscr{B}(n, k)$ is linearly equivalent to the arrangement of hyperplanes spanned by subsets of $\left\{w_{1}, \ldots, w_{n}\right\}$ of size $\ell-1$. 
The proof of 2.2 will occupy the remainder of this section.

Remark 2.3 (Ziegler). In the language of matroid theory (oriented if $\mathbf{K}=\mathbf{R}$ ), Theorem 2.2 asserts that the arrangement $\mathscr{B}(n, k)$ realizes an adjoint of the matroid determined by the vector configuration $\left\{w_{1}, \ldots, w_{n}\right\}$, which in turn is the dual of that determined by $\overline{\mathscr{A}}_{0}$ [2].

Let $\mathscr{A}$ be an affine arrangement in $\mathbf{K}^{k}$ such that some $k$ of the elements of $\mathscr{A}$ have nonempty intersection. Suppose the hyperplanes of $\mathscr{A}$ are given by nonhomogeneous defining equations $\phi_{i}(x)+\alpha_{i}=0$, with $\alpha=\left(\alpha_{1}, \ldots, \alpha_{n}\right) \in$ $\mathbf{K}^{n}$. We associate to $\mathscr{A}$ a $k$-dimensional affine subspace $P(\mathscr{A})$ of $\mathbf{K}^{n}$, the image of the map $\left(\phi_{1}, \ldots, \phi_{n}\right)+\alpha: \mathbf{K}^{k} \rightarrow \mathbf{K}^{n}$. Note that $P(\mathscr{A})$ is parallel to the linear subspace $P(\overline{\mathscr{A}})$; in fact, $P(\mathscr{A})=\alpha+P(\overline{\mathscr{A}})$. The affine arrangement $\mathscr{A}$ can be recovered from $P(\mathscr{A})$ given a basis for $P(\overline{\mathscr{A}})$ and a point $\alpha$ of $P(\mathscr{A})$ to serve as the origin.

Note that $\mathscr{A}$ is a general position arrangement if and only if $P(\mathscr{A}) \cap \xi_{I}$ is empty for $|I|=k+1$ and is a single point if $|I|=k$.

Remark 2.4. Let $\widehat{\Gamma}(n, k)$ denote the space of $k$-dimensional affine subspaces $P$ of $\mathbf{K}^{n}$ satisfying $P \cap \xi_{I}=\varnothing$ for $|I|=k+1$ and $\left|P \cap \xi_{I}\right|=1$ for $|I|=k$. This space is denoted by $V(n, k)$ in [6, Remark 3]. There is a homeomorphism between $\Gamma(n+1, k+1)$ and $\widehat{\Gamma}(n, k)$, obtained by intersecting elements of $\Gamma(n+1, k+1)$ with the hyperplane $x_{n+1}=1$. The map $P(\mathscr{A}) \mapsto P(\overline{\mathscr{A}})$ carries $\widehat{\Gamma}(n, k)$ to $\Gamma(n, k)$, by translating affine subspaces to the origin. The composition of these two maps yields a projection $\Xi: \Gamma(n+1, k+1) \rightarrow \Gamma(n, k)$ of the generic strata. As we will see below, the fiber over $P(\overline{\mathscr{A}})$ coincides with the complement of the essential part of the discriminantal arrangement associated with $\mathscr{A}$. The example of the next section will show that the homotopy type of the fiber is not constant, so the map $\Xi$ is not a fibration.

Lemma 2.5. Suppose $\overline{\mathscr{A}}$ is generic, and $|\overline{\mathscr{A}}|=|\mathscr{A}|$. Then $P(\mathscr{A}) \cap \xi_{I}$ consists of a single point if $|I|=k$.

Proof. By the hypothesis $|\overline{\mathscr{A}}|=|\mathscr{A}|$ the hyperplanes $H_{i} \in \mathscr{A}$ with $i \in I$ translate to $k$ distinct hyperplanes in $\overline{\mathscr{A}}$. Since $\overline{\mathscr{A}}$ is generic, these hyperplanes intersect only at the origin. So $P(\overline{\mathscr{A}}) \cap \xi_{I}=0$. Then $P(\mathscr{A}) \cap \xi_{I}$ is either empty or a single point. Suppose $P(\mathscr{A}) \cap \xi_{I}=\varnothing$. Choose $I_{0} \subseteq I$ maximal with $P(\mathscr{A}) \cap \xi_{I_{0}} \neq \varnothing$. Note $I_{0} \neq \varnothing$ since $P(\mathscr{A}) \cap \xi_{\{i\}} \neq \varnothing$ for $1 \leq i \leq n$. By assumption $\left|I_{0}\right|<k$. Then the codimension in $P(\mathscr{A})$ of $P(\mathscr{A}) \cap \xi_{I_{0}}$ is less than $k$, so $\operatorname{dim}\left(P(\mathscr{A}) \cap \xi_{I_{0}}\right) \geq 1$. Also $P(\mathscr{A}) \cap \xi_{I_{0}}$ is parallel to $\xi_{I \backslash I_{0}}$ by maximality of $I_{0}$. Then there exist distinct $x, y \in P(\mathscr{A}) \cap \xi_{I_{0}}$, which will satisfy $0 \neq x-y \in P(\overline{\mathscr{A}}) \cap \xi_{I_{0}} \cap \xi_{I \backslash I_{0}}=P(\overline{\mathscr{A}}) \cap \xi_{I}$. This provides a contradiction.

We now complete the proof of Theorem 2.2. By definition $U(n, k)$ is the set of general position arrangements $\mathscr{A}$ satisfying $\overline{\mathscr{A}}=\overline{\mathscr{A}} 0$. By $2.5 \mathscr{A} \in$ $U(n, k)$ if and only if $\overline{\mathscr{A}}=\overline{\mathscr{A}_{0}}$ and $P(\mathscr{A}) \cap \xi_{I}=\varnothing$ for $|I|=k+1$. By choosing linear forms $\phi_{i}$ for $\mathscr{A}_{0}$ we identify the arrangements $\mathscr{A}$ satisfying $\overline{\mathscr{A}}=\mathscr{\mathscr { A }}_{0}$ with points $\alpha \in \mathbf{K}^{n}$. Recall that $P=P\left(\overline{\mathscr{A}_{0}}\right)$ and $Q$ is the orthogonal complement of $P ; P(\mathscr{A})$ is parallel to $P$. We write $\alpha=\beta+\gamma$ 
with $\beta \in P$ and $\gamma \in Q$. The location of $P(\mathscr{A})$ is uniquely determined by $\gamma$, independent of $\beta$. Thus the condition $\mathscr{A} \in U(n, k)$ imposes some restrictions on $\gamma$, but not on $\beta$. So we see that $U(n, k)$ is the cartesian product of $P \cong \mathbf{K}^{k}$ with a subspace (in the topological sense) of $Q$. It remains only to show that this subspace is isomorphic to the complement of the essential arrangement described in 2.2 .

To carry out the identification of $Q$ with $\mathbf{K}^{\ell}$ we assume without loss that $\left\{v_{1}, \ldots, v_{\ell}\right\}$ is an orthonormal basis for $Q$. Let us write $\beta=\sum_{i=1}^{\ell} \beta_{i} v_{i}$. Then $x \in P(\mathscr{A})$ if and only if $\sum_{j=1}^{n} v_{i j} x_{j}=\beta_{i}$ for each $i, 1 \leq i \leq \ell$. The intersection $P(\mathscr{A}) \cap \xi_{I}$ is nonempty if and only if this linear system is consistent when $x_{i}$ is set equal to zero for $i \in I$. This occurs if and only if $\beta$ lies in the span of the set $\left\{w_{j} \mid j \notin I\right\}$. Thus $\mathscr{A} \in U(n, k)$ iff $P(\mathscr{A}) \cap$ $\xi_{I}=\varnothing$ for $|I|=k+1$ iff $\beta$ is not in the span of any $\ell-1$ of the vectors $\left\{w_{1}, \ldots, w_{n}\right\}$. So $U(n, k)$ is the product of $\mathbf{K}^{n-k}$ with the complement of the arrangement of hyperplanes spanned by subsets of $\left\{w_{1}, \ldots, w_{n}\right\}$ of size $\ell-1$. This completes the proof of Theorem 2.2 .

Remark 2.6. It is not hard to see that the mapping $\Xi: \Gamma(n+1, k+1) \rightarrow$ $\Gamma(n, k)$ defined in Remark 2.4 carries a generic arrangement of $n+1$ hyperplanes in $\mathbf{K}^{k+1}$ to its contraction (or restriction) on the $(n+1)^{s t}$ hyperplane [8, Definition 1.13]. There is another map of generic strata $\Delta: \Gamma(n+1, \ell) \rightarrow$ $\Gamma(n, \ell)$ obtained by deletion of the $(n+1)^{s t}$ hyperplane. (This is the map used, for example, in [4].) The fiber of $\Delta$ is easily seen to be the adjoint of a general position configuration of points, as defined in the next paragraph. Indeed, to result in a general position arrangement, the normal vector for $(n+1)^{s t}$ hyperplane must lie in the complement of the hyperplanes spanned by the normals to the first $n$ hyperplanes. Our main Theorem 2.2 shows that this fiber is isomorphic to the complement of the essential part of a discriminantal arrangement, the fiber of the contraction mapping $\Xi$. This is not a coincidence. In fact, deletion and contraction are dual operations, and these two maps $\Xi$ and $\Delta$ (where $k+\ell=n$ ) are connected by the duality mapping $P \mapsto P^{\perp}$ of the corresponding Grassmannians, as illustrated in the following diagram:

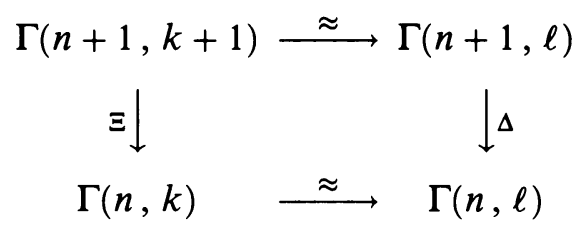

\section{VARIATION OF COMBINATORIAL TYPE}

Let $\Lambda=\left\{w_{1}, \ldots, w_{n}\right\}$ be a vector configuration in $\mathbf{K}^{\ell}$. Let $\mathscr{A}(\Lambda)$ be the arrangement of hyperplanes spanned by subsets of $\Lambda$. We call $\mathscr{A}(\Lambda)$ the adjoint of $\Lambda$. According to [1] the underlying matroid of $\mathscr{A}(\Lambda)$ is an adjoint of the matroid determined by $\Lambda$.

Theorem 2.2 states that $\mathscr{B}(n, k)_{\text {ess }}=\mathscr{A}(\Lambda)$ for a particular general position configuration $\Lambda$. Its proof has the following easy corollary. 
Proposition 3.1. Let $\Lambda$ be an arbitrary general position configuration of $n$ points in $\mathbf{K}^{\ell}$. Then there exists an arrangement $\mathscr{A}_{0}$ in general position in $\mathbf{K}^{n-\ell}$ such that the associated discriminantal arrangement has essential part linearly equivalent to $\mathscr{A}(\Lambda)$.

Proof. Write $\Lambda=\left\{w_{1}, \ldots, w_{n}\right\}$, with $w_{j}=\left(w_{1 j}, \ldots, w_{\ell j}\right) \in \mathbf{K}^{\ell}$. Let $P$ be the null space of the matrix $\left[w_{i j}\right]$. Then $P$ is a $k$-dimensional subspace of $\mathbf{K}^{n}$, $k=n-\ell$. Choose a basis $\left\{b_{1}, \ldots, b_{k}\right\}$ for $P$, with $b_{j}=\left(b_{1 j}, \ldots, b_{n j}\right)$. Then the linear forms $\phi_{i}$ defining $\overline{\mathscr{A}}_{0}$ are given by $\phi_{i}(x)=\sum_{j=1}^{k} b_{i j} x_{j}$. Since $\Lambda$ is in general position $\overline{\mathscr{A}_{0}}$ is a generic arrangement.

Note that $\left\{v_{1}, \ldots, v_{\ell}\right\}$ is a basis for $P^{\perp}$, where $v_{i}=\left(w_{i 1}, \ldots, w_{i n}\right)$; we may assume without loss that this basis is orthonormal.

Choose a point $\beta=\left(\beta_{1}, \ldots, \beta_{\ell}\right)$ in the complement of the arrangement $\mathscr{A}(\Lambda)$, and let $\alpha=\sum_{i=1}^{\ell} \beta_{i} v_{i}$. Then the affine hyperplanes given by $\phi_{i}(x)+$ $\alpha_{i}=0,1 \leq i \leq n$, form the desired arrangement $\mathscr{A}_{0}$.

Consider now the case $\mathbf{K}=\mathbf{R}$ and $n=k+3$. According to 3.1 we may choose any collection of $n$ points $w_{1}, \ldots, w_{n}$ in general position in $\mathbf{R}^{3}$, and the $\left(\begin{array}{l}n \\ 2\end{array}\right)$ planes spanned by pairs of these vectors will form the essential part of a discriminantal arrangement "of type $(n, n-3)$ ". But these arrangements will have different intersection lattices depending on the placement of the $w_{j}$. In the context of oriented matroids the following example appears in [1].

Example 3.2. Let $\Lambda_{1}$ be the set of vertices of a regular hexagon in the plane $x_{3}=1$ in $\mathbf{R}^{3}$. Let $\Lambda_{2}$ be a set of six points in general position in the plane $x_{3}=1$ such that no two of the segments joining them are parallel. Then $\mathscr{A}\left(\Lambda_{1}\right)$ and $\mathscr{A}\left(\Lambda_{2}\right)$ each consist of 15 planes. But the intersection lattices differ. In $\mathscr{A}\left(\Lambda_{2}\right)$ there are six lines of multiplicity five, two on each plane, and 45 lines of multiplicity two, three on each plane. This is precisely the combinatorial structure described by Manin and Schechtman in subsection 7 of [6]. But in $\mathscr{A}\left(\Lambda_{1}\right)$ there are lines of multiplicity three, formed by the "diagonals" of the hexagon. Thus $\mathscr{A}\left(\Lambda_{1}\right)$ and $\mathscr{A}\left(\Lambda_{2}\right)$ have nonisomorphic intersection lattices.

The procedure of 3.1 is quite easy to carry out for the configuration $\Lambda_{1}$ using Mathematica. The defining forms for $\overline{\mathscr{A}_{0}}$ are

$$
\begin{aligned}
2 x_{1}+2 x_{2}+x_{3} & =0 \\
2 x_{1}+3 x_{2}+2 x_{3} & =0 \\
x_{1}+2 x_{2}+2 x_{3} & =0 \\
x_{3} & =0 \\
& =0 \\
x_{2} & =0
\end{aligned}
$$

The base arrangement $\mathscr{A}_{0}$ is obtained by replacing the right-hand side with a suitable nonzero vector to obtain a general position affine arrangement in $\mathbf{R}^{3}$.

The three diagonals of the hexagon correspond to the hyperplanes

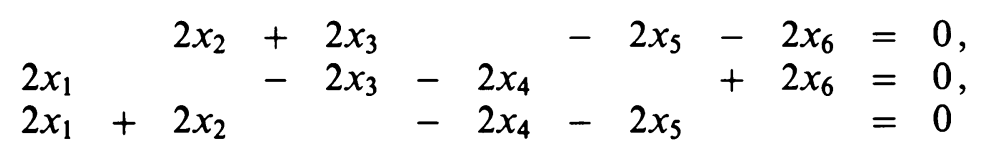


in $\mathscr{B}(n, k)$, labeled $(14),(25)$, and (36) in the notation of [6, 7]. It is easy to check that $(14) \cap(25) \subseteq(36)$ as claimed. This contradicts the assertion of [6, Proposition 4(c)] that $D_{J_{1}} \cap D_{J_{2}} \subseteq D_{J}$ implies $J=J_{1}$ or $J=J_{2}$. Here $(i, j)$ denotes $D_{J}$ for $J=\{1, \ldots, 6\} \backslash\{i, j\}$. All the assertions in the proof of Proposition 4 of [6] hold for trivial reasons in this case.

We conclude by pointing out that the arrangements $\mathscr{A}\left(\Lambda_{1}\right)$ and $\mathscr{A}\left(\Lambda_{2}\right)$ will also have different Poincare polynomials. So when the complexifications are considered as discriminantal arrangements over $\mathbf{K}=\mathbf{C}$, the complements have different betti numbers. Thus homotopy type is also dependent on the original $\mathscr{A}_{0}$.

\section{ACKNOWLEDGMENTS}

This result was discovered in the days surrounding a brief visit to the University of Iowa. I am grateful to Dick Randell for his hospitality and helpful comments. I also benefited from discussions at various points with Günter Ziegler, Boris Shapiro, and Dick Hain.

\section{REFERENCES}

1. A. Bachem and W. Kern, Adjoints of oriented matroids, Combinatorica 6 (1986), 299-308.

2. A. Björner, M. Las Vergnas, B. Sturmfels, N. White, and G. Ziegler, Oriented matroids, Cambridge Univ. Press, Cambridge, 1992.

3. I. M. Gelfand, R. M. Goresky, R. D. MacPherson, and V. V. Serganova, Combinatorial geometries, convex polyhedra, and Schubert cells, Adv. Math. 63 (1987), 301-316.

4. R. Hain and R. MacPherson, Higher logarithms, Illinois Math. J. 34 (1990), 392-475.

5. R. Lawrence, A presentation for Manin and Schechtman's higher braid groups, MSRI preprint series \#04129-91, April, 1991.

6. Y. I. Manin and V. V. Schechtman, Arrangements of hyperplanes, higher braid groups and higher Bruhat orders, Algebraic Number Theory - in honor of K. Iwasawa (Amsterdam), Adv. Stud. in Pure Mathematics, vol. 17, North-Holland, Amsterdam, 1989, pp. 289-308.

7. P. Orlik, Introduction to arrangements, CBMS Regional Conf. Ser. in Math., vol. 72, Amer. Math. Soc., Providence, RI, 1989.

8. P. Orlik and H. Terao, Arrangements of hyperplanes, Springer-Verlag, Berlin, Heidelberg, and New York, 1992.

Department of Mathematics, Northern Arizona University, Flagstaff, Arizona 86011-5717

E-mail address: mjf@caliban.math.nau.edu 